Winter Meeting - Joint meeting between the Nutrition Society and the Royal Society of Medicine, 11-12 December 2012,

Dietary Strategies for the Management of Cardiovascular Risk

\title{
Exploring the myth: Does eating celery result in a negative energy balance?
}

\author{
M. E. Clegg and C. Cooper \\ Functional Food Centre, Department of Sport and Health Sciences, Oxford Brookes University, Gipsy Lane, Oxford, \\ $O X 3 O B P, U K$
}

With the prevalence of obesity increasing across the world, there is growing demand for effective strategies to reduce the severity of the problem and moderate risks for developing other associated diet-induced diseases. Celery is a readily available whole-food that has the ability to add bulk and flavour to a meal, without adding excess calories. Celery is also subject to a renowned health myth, that when consuming celery there is a 'negative' intake of calories and therefore the energy required for its digestion, assimilation and nutrient storage is assumed to be greater than the energy it itself contains. The aim of the current study is to explore the diet induced thermogenesis (DIT) associated with celery consumption.

Fifteen healthy female volunteers (age: 23.5 (SEM 0.6) yr; height: 1.67 (SEM 0.02) m; weight: 59.6 (1.8 SEM) kg) came to the laboratory for testing following an overnight fast. In the laboratory they rested for 30 minutes in a supine position before resting metabolic rate (RMR) was measured for 30 minutes using an indirect calorimeter. During the last 10 minutes of the 30 minute rest period, the ventilated-hood of the indirect calorimetry system was placed over subject's heads to acclimatise them to the hood environment. Following the 30 minutes RMR measurements, whilst still under the hood and measurements were being taken, the volunteers then consumed $100 \mathrm{~g}$ of celery $\left(16 \mathrm{kcal}^{(1)}\right.$ in 10 minutes. For the subsequent 180 minutes, DIT was measured at regular $30-$ minute intervals for 20 minutes. Throughout the assessment, subjects rested quietly but stayed awake. Energy expenditure and substrate oxidation were calculated using the equations developed by Lusk ${ }^{(2)}$.

RMR varied considerably between volunteers, ranging from 1283.56 to $1893.69 \mathrm{kcal} / \mathrm{day}$, with a mean value of 1657 (sEM 40 ) $\mathrm{kcal} / \mathrm{day}$. During the first 20 minute DIT measurements, all subjects experienced an increase in EE and fat oxidation: mean increase 0.15 (SEM 0.02 ) and 0.08 (SEM 0.01) kcal/min, respectively. Total DIT was 13.76 (SEM 2.65) kcal and total fat oxidation was 8.31 (SEM 1.76) kcal. From the consumption of a $16 \mathrm{kcal}(100 \mathrm{~g})$ sample of celery, $13.76 \mathrm{kcal}$ were expended in total.

In conclusion, it was found that the consumption of celery $(16 \mathrm{kcal})$ did not induce a negative energy balance in healthy women. However the DIT of $100 \mathrm{~g}$ of celery was $86 \%$ of the total energy intake. This fact combined with the high fibre and water content of celery does make it a good snack for inclusion in a diet for weight loss or management.

1. USDA National Agricultural Library (2012) National Nutrient Database for Standard Reference, Release 24

2. Lusk G (1928) The Elements of the Science of Nutrition. Philadelphia, PA: WB Saunders Co. 